\title{
Interfacing for the SKED system
}

\author{
ROBERT I. COBEZ and ARTHUR G. SNAPPER \\ Westerm Michigan University, Kalamazoo, Michigan 49008
}

\begin{abstract}
Four new interface modules, a new input module, a duration measuring module, an omnibus module, and a high-speed reader module, have been developed for the SKED interface to further optimize the use of the small computer in the laboratory.
\end{abstract}

The role of computers has been expanded in such a way that they are no longer just data processors. Computers have been connected to external devices in such a manner as to enable the computer to monitor and control the functions of these devices. To permit the interaction of computer and external machine, an interface is used. The interface essentially functions as a translator of signals from the computer to the external machine and from the external machine back into the computer. Computer signals, which generally lack sufficient power to drive external devices, are sent into the interface to be transformed into more powerful signals which are subsequently sent to drive the external devices, such as relays. Operating signals from external devices, returning to the computer, are attenuated by the interface into signals that can be understood and processed by the computer.

Small computers (minicomputers) are being utilized in the laboratory for data acquisition and control in ever increasing numbers because of their low cost, their ease of operation, and their capabilities for being interfaced with various machines. Naturally, there are a variety of computer systems to choose from, each of which has its advantages and disadvantages.

The SKED system described by Snapper and Kadden (1973) and interfacing developed by State System, Inc., ${ }^{1}$ has proven to be highly reliable and economical for data acquisition and control of behavioral experiments in the laboratory. This system consists of a powerful programming software package, SKED, to program any experiment desired and an interface which connects 12 experimental stations to any PDP $-8^{2}$ series minicomputer.

Three basic integrated circuit modules, input, output, and clock, along with indicator lights, relays, and pushbuttons mounted on standard relay panels comprise the SKED interface. Responses, such as barpresses or keypecks, from the experimental chambers energize relays which send signals into the input module of the interface, where they are converted into signals that can be received by the computer. Programmed signals from the computer are sent into the output module of the interface through which these signals drive relays that control the various devices, tones, lights, shocks, inside the experimental chamber. A dual-speed clock $(1 \mathrm{msec}$ and

This work was supported in part by Research Scientist Development Award K2-MH-70483 from the National Institute of Mental Health. We would like to thank Nelia Masiques for her assistance in the preparation of the figures.
$10 \mathrm{msec}$ ) emits timing pulses that regulate the speed of programmed events from the computer and subsequent retrieval and processing of signals from the experimental stations. Programmed signals from the computer and responses from the chambers illuminate indicator lights mounted on standard size rack panels, thereby providing a simple and efficient means to monitor the status of currently active chambers. Butler (1974) has presented a more detailed description of the operation and hardware utilized in the SKED interface in an earlier paper.

Four new interface modules have been developed by State Systems for the SKED interface to further enhance computerized control of laboratory experiments. They include a newly designed input integrated circuit module, a duration module, a complete interface module, the omnibus option, and a module to interface a high-speed paper-tape reader with the PDP-8 series computers.

A new input integrated circuit module which processes response signals from the experimental stations has been developed. Many of the components that were present in the former input module have been eliminated on the new one or replaced by integrated circuit chips. This improvement has increased the efficiency of the new input module and is especially beneficial because of the increased ease in trouble-shooting electrical problems.

A simplified logic drawing of the new input module is presented in Figure 1. Basically, a response on the operandum $(0)$ in the experimental chamber or a closure of the pushbutton (PB) by the experimenter will energize a relay, causing the two separate commons of a doublepole double-throw relay to switch to their respective normally open (NO) contacts. A light on the relay panel indicates the activation of the relay. When energized, the relay also causes the level of the signal on the upper AND gate in Figure 1 to change from a logical zero to a logical one. The SKED interface operates on positive logic. When the input module is enabled by the proper device code, and when the proper input-output pulse (IOP) is generated, and when a response from the experimental station occurs, all happening simultaneously, a signal is transmitted into a specific bit in the accumulator $(\mathrm{AC})$ and is subsequently processed by the SKED software.

Like the previous input module, each new input module can handle 36 separate response lines from the experimental chambers into the computer. Each line can be assigned to a different operandum in the chamber. 


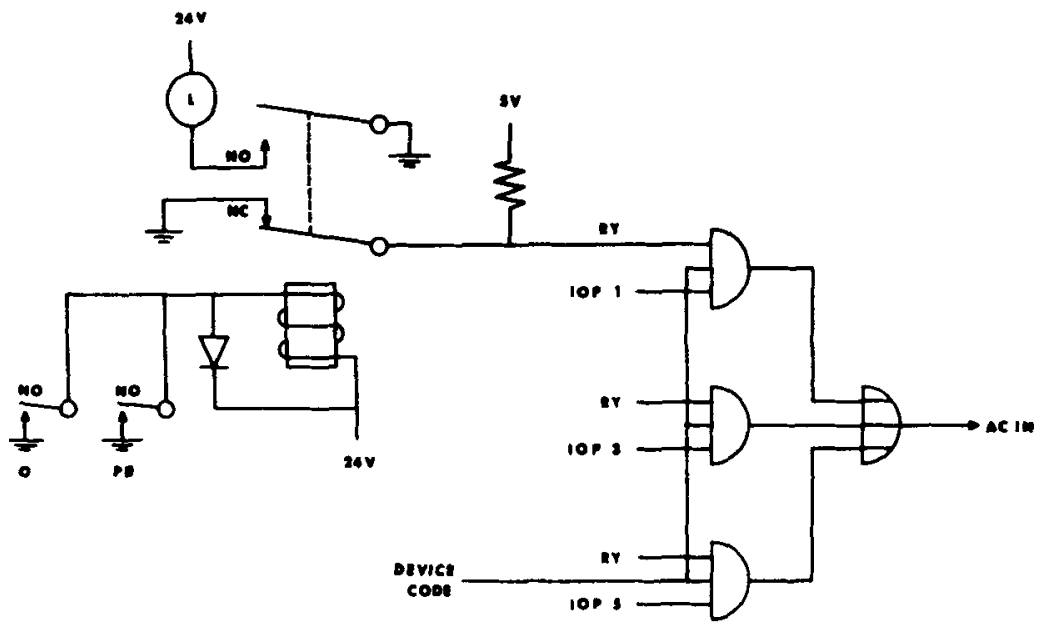

Figure 1. Simplified logic drawing of the new input in tegrated circuit module.

Each set of 12 input lines constitutes a bufter and is controlled by a separate IOP. Also, each line of each buffer is assigned to a specific accumulator bit, of which there are 12 , thereby accommodating all 36 individual response lines of the input module. Signals caused by responses or pushbutton closures are channeled into a multiplexer, represented by the three parallel AND gates in Figure 1. To conserve space on the input module and to increase efficiency, the multiplexer is used to channel signals from different buffers into the same accumulator bit at different times. The multiplexed signal drives a transistor, which can drive $100 \mathrm{~mA}$ of current, causing a logical zero to be generated by the transistor. This signal is subsequently inverted when it finally enters the accumulator. An experimental station can have any number of input or output lines assigned to it.

Another interfacing module developed by State Systems and used in a recent experiment by Schwartz, Hamilton, and Silberberg (1975) permits the precise measurement of response durations of $1 \mathrm{msec}$ or more. The circuitry for this module is mounted on a standard Digital Equipment Corporation (DEC) W-966 wire-wrap card. This module can be connected with six individual operanda at one time.

The basic logic of the duration circuit is shown in Figure 2 and can be thought of in terms of an exclusive OR circuit. The experimental operandum is connected directly with the integrated circuitry of the duration module. This avoid, the usual 10- to 20-msec delay caused by relay-activated signals. As can be seen in Figure 2, two separate lines are connected to the computer from the single input line of the operandum. Until

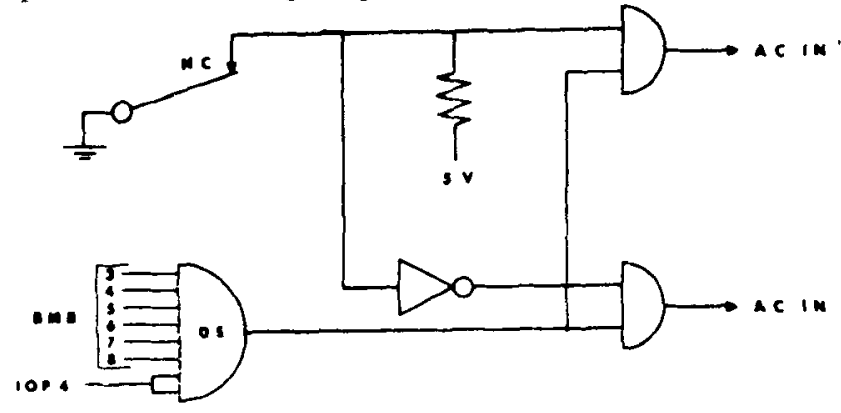

Figure 2. Simplified logic drawing of the duration module. a response (such as a leverpress) occurs, a logical zero will be present on the response line of the upper AND gate. Also, a logical zero will appear in the accumulator bit connected with the upper gate. However, the lower AND gate has a logical one present on its response line and in the specific accumulator bit associated with it. The duration circuit uses the same type of transistor configuration as the input module does to send signals into the accumulator. When the duration card is enabled by the proper device code on the device selector (DS), a response will cause a logical one to be produced by the upper AND gate, resulting in a logical one appearing in the accumulator bit associated with it. In addition, the output of the lower AND gate will change from a logical one to a logical zero, as will the level of the signal in the accumulator bit associated with the lower AND gate.

Typically, in the study of small durations, the experimenter will be running with the 1 -msec clock enabled, which means that the status of each experimental chamber is examined and processed every $1 \mathrm{msec}$. Therefore, the near-instantaneous switching of the accumulator bits, from zero to one and one to zero, can be readily detected to yield precise measures of response durations.

For those laboratories with a limited budget and a desire for a small interface, an inexpensive interface module has been developed that combines all of the circuitry of the standard input, output, and clock modules. The omnibus interface module has 12 separate input lines and 24 separate output lines along with an adjustable clock. For those laboratories that have PDP-8/A computers, an omnibus interface can be obtained with. out the clock circuitry. All of the integrated circuitry for this interface is wired on a single DEC M1709 card and fits normally into any one row in the omnibus of any PDP-8/A, E, F, or $M$ minicomputer. It does not require the KA8-E option for operation. All that is necessary to complete this interface is the relays, indicator lights, and pushbuttons mounted on the rack panels.

The major advantage of this interface, besides being inexpensive (less than $\$ 1,000$ ) is that the integrated circuitry fits directly into the user's computer and eliminates having to buy separate input, output, and clock modules along with a busall, the device used to hold the 

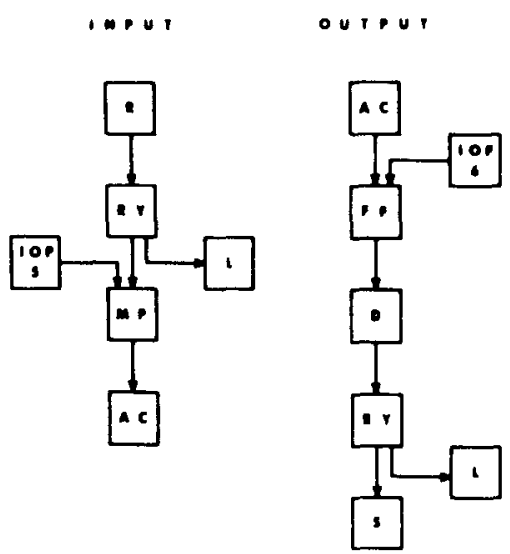

modules. However, the major disadvantage of this system is that no expansion beyond the 12 input lines and 24 output lines is possible. To expand, the user would have to purchase the standard SKED interface or possibly another omnibus module.

The block drawing presented in Figure 3 provides a simplified overview of the basic sections of the omnibus module: input, output, and clock. The input circuitry for the omnibus module is similar to that incorporated in the input module. Thus, a response ( $R$ ) activates a relay (RY) which enables an indicator light (L) and sends a signal into a multiplexer (MP), which, when gated with the proper IOP, transmits a signal into the proper accumulator bit (AC).

When a programmed SKED instruction activates some type of output stimulus in the experimental chamber. such as a houselight or a tone, a logical one will appear in the accumulator bit $(\mathrm{AC})$ corresponding to the stimulus. The logical one is output from the accumulator and sets a flip-flop (FF). When the proper IOP occurs, a signal from the flip-flop enables a relay driver (D) which is capable of driving $30 \mathrm{~V} \mathrm{dc}$ at $40 \mathrm{~mA}$. This boosted signal energizes a relay (RY) which activiates the stimulus $(\mathrm{S})$ in the chamber and an indicator light (L).

Clock pulses are generated by two one-shots (OS), monostable multivibrators, triggering each other. Timing can be adjusted with the variable resistor $(\mathrm{T})$. The output of the first one-shot also causes a logical one to be produced from the flip-flop beneath it by enabling the clock pulse (CK) line of that flip-flop. The output from the flip-flop is an interrupt request (IR) and, when gated $(G)$ with an IOP, will cause a skip (SKP) to be generated in the software. Another IOP (IOP 2) can clear the signal from the flip-flop. An IOP 3 on the clock pulse (CK) line of the lower flip-flop in Figure 3 will result in the interrupt enable (IE) line becoming active. If the interrupt enable line and interrupt request lines are both active, an interrupt (INT) occurs in the computer software. This flip-flop is cleared by an IOP 0 .

Voltage changes in the computer, caused by the addition or deletion of cards in the computer's omnibus, will necessitate periodic adjustments in the clock circuit. The one-shot clock has an accuracy range of $1 \%$.
Data transfer into the computer has been facilitated by the incorporation of a high-speed reader interface, for the Addmaster high-speed general-purpose paper-tape reader. This reader accepts the standard eight-channel paper tape used in the ASR-33 Teletype and can be purchased from the manufacturer, Addmaster Corporation, ${ }^{3}$ or from State Systems. A DEC W966 wire-wrap card holds the reader circuitry. The high-speed reader, when coupled with the reader interface, can reliably transfer 110 characters/sec (CPS), which is quite an improvement over the 10 CPS with the ASR-33 Teletype. In fact, the time saved by using this high-speed reader makes it a very worthwhile investment.

The interface modules discussed above, with the exception of the input module, are supplemental to the standard SKED interface. With the exception of the omnibus module, all other modules discussed fit in the standard SKED busali. These modules are inexpensive and very reliable in operation. Their utilization in the computerized laboratory will greatly increase the range of options available to the experimenter for data acquisition, processing, and control.

\section{REFERENCES}

ButLER, F. The BUS-ALL: An inexpensive set of interface for the SKED system. Behavior Research Methods \& Instrumen tation. 1974, 6, 171-173.

Schwartz, B.. Hamilton, B., \& Sillerburg, A. Behavioral contrast in the pigeon: A study of the duration of key pecking maintained on multiple schedules of reinforcement. Journal of the Experimental Analysis of Behavior. 1975, 24, 199-206.

SNAPPER, A., \& KADDEN, R. Time-sharing in a small computer based on a behavioral notational system. In B. Weiss (Ed.), Digital computers in the behavioral laboratory. New York: Appleton-Century-Crofts. 1973.

\section{NOTES}

1. State Systems, Inc., P. O. Box 2215, Kalamazoo, Michigan 49003

2. PDP is a registered trademark of Digital Equipment Corporation, Maynard, Massachusetts.

3. Addmaster Corporation, 416 Junipero Serra Drive, San Gabriel, California 91776. 